\title{
Baixo peso ao nascer e proficiência motora em crianças: uma revisão sistemática
}

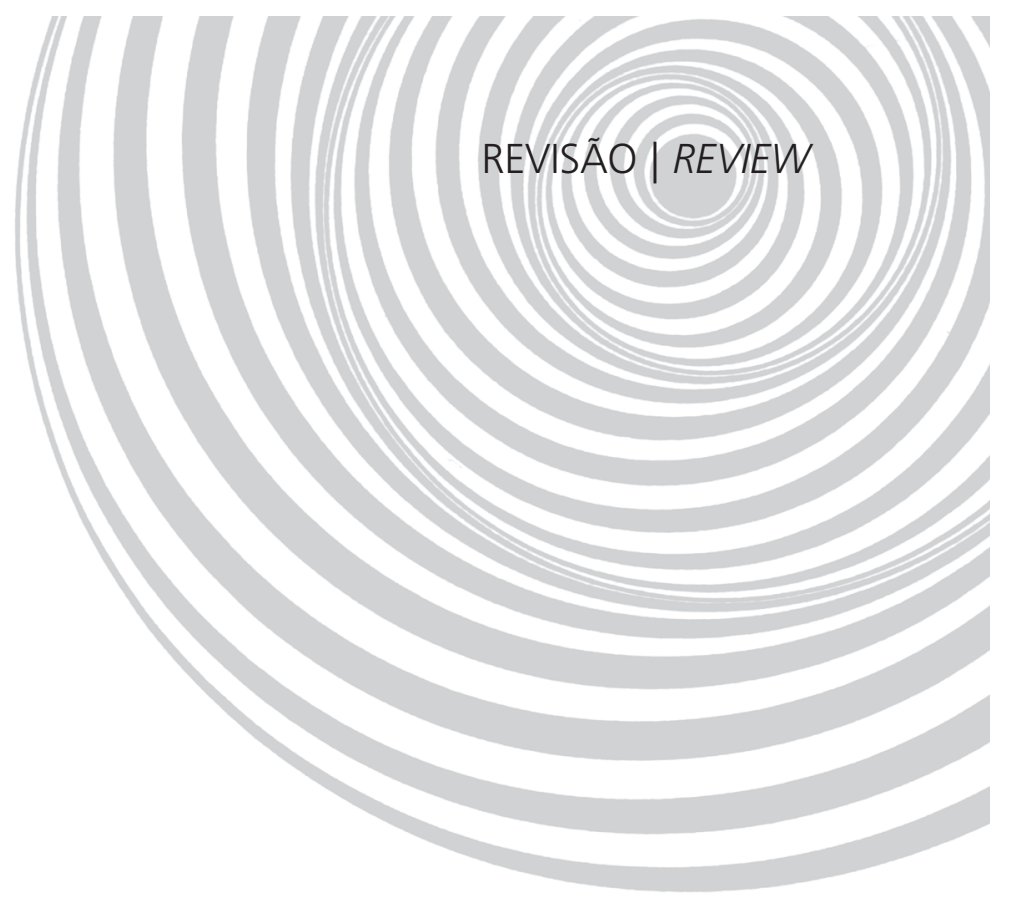

\author{
Low birth weight and motor proficiency \\ in children: a systematic review
}

Aline Kety Maria de SIQUEIRA ${ }^{1}$

Carol Góis LEANDRO'

RE S U M O

O objetivo deste trabalho foi analisar estudos que associam o baixo peso ao nascer e a proficiência motora em crianças com idade entre um e dez anos. A revisão sistemática da literatura foi realizada em base de dados eletrônica e lista de referência dos artigos publicados. Foram utilizados os seguintes descritores na língua inglesa: "infant low birth weight", "infant very low birth weight", "infant extremely low birth weight", "motor skills", "psychomotor performance", "child development", "human development", "growth \& development", "growth" e "fetal development". A análise dos estudos envolveu leitura de títulos, resumos e textos completos. No total, 18 artigos foram lidos na íntegra; apenas dois estudos tiveram delineamento longitudinal; e os demais estudos eram transversais. As crianças avaliadas tinham idade entre 9 meses e 9 anos. Na avaliação do desenvolvimento motor, há ampla utilização de instrumentos já padronizados, que avaliam habilidades motoras grossas e finas de maneira quantitativa. Os resultados dos estudos apontam que crianças nascidas com peso abaixo de $2.500 \mathrm{~g}$ apresentam algum tipo de deficit motor. As habilidades mais afetadas foram as habilidades motoras finas e o equilíbrio. O peso ao nascer é uma variável que deve ser considerada nos estudos sobre o desenvolvimento de habilidades motoras básicas das crianças.

Termos de indexação: Crescimento e desenvolvimento. Criança. Destreza motora. Peso ao nascer.

A B S T R A C T

The main goal of the present study was to review studies that associate low birth weight and motor proficiency in children aged 1 to 10 years. An electronic database and reference lists of the published papers were used for collecting articles and a systematic review of the literature was done. The following keywords were used:

\footnotetext{
1 Universidade Federal de Pernambuco, Centro Acadêmico de Vitória, Núcleo de Educação Física e Ciências do Esporte. R. Alto do Reservatório, s/n., Bela Vista, 55608-680, Vitória de Santo Antão, PE, Brasil. Correspondência para/Correspondence to: AKM SIQUEIRA. E-mail: <alinsiq@hotmail.com>.
} 
"infant low birth weight", "infant very low birth weight", "infant extremely low birth weight", "motor skills", "psychomotor performance", "child development", "human development", "growth \& development", "growth" and "fetal development". Study analysis included reading the titles, abstracts and manuscripts. Of a total of 18 papers, only two had a longitudinal design while 16 had a cross-sectional design. The children were aged between 9 months and 9 years. Motor development was assessed by using standardized instruments for assessing fine and gross motor skills in a quantitative way. The studies showed that low birth weight children (weighing less than 2,500g) have some type of motor deficit. The most affected skills were fine motor skills and balance. Birth weight should be considered an important variable in studies on the development of basic motor skills in children.

Indexing terms: Growth and development. Child. Motor skill. Birth weight.

\section{N T R O D U ÇÃ O}

O crescimento fetal é o resultado da interação entre o genoma do próprio feto e do meio ambiente intrauterino. Essa interação parece indicar a existência de uma plasticidade durante o desenvolvimento ${ }^{1-3}$. No sentido mais estrito, a plasticidade durante o desenvolvimento é entendida como a propriedade de um determinado genótipo produzir diferentes fenótipos em resposta a distintas condições do ambiente ${ }^{4}$. O feto humano responde à desnutrição, à exposição a hormônios (glicocorticoides)5,6, à ação de fármacos (antidepressivos) ${ }^{7}$, à disfunção placentária e a outras influências adversas, alterando a trajetória de seu desenvolvimento e diminuindo o crescimento ${ }^{8}$.

O reduzido crescimento corporal no nascimento não é visto como causador de consequências a longo termo, mas como marcador de uma resposta adaptativa fetal para uma limitação no ambiente intrauterino, resultando em mudanças nos tecidos e órgãos em desenvolvimento ${ }^{9}$. As mudanças, necessariamente, não se evidenciam no nascimento, mas podem resultar em respostas perturbadas na vida mais tarde 9 . Nesse sentido, o baixo peso ao nascer pode ser entendido como marcador de alguma consequência adversa no ambiente fetal ${ }^{8}$.

A World Health Organization ${ }^{10}$ classifica as crianças em três categorias quanto ao peso de nascimento: Peso Normal ao Nascer (PNN), com um peso maior que 2.500g; Baixo Peso ao Nascer (BPN), com um peso menor que 2.500g; Muito Baixo Peso ao Nascer (MBPN), com um peso me- nor que 1500g; Extremo Baixo Peso ao Nascer (EBPN), com um peso menor que $1.000 \mathrm{~g}^{10}$. Tomando como base o peso ao nascer, recentes estudos longitudinais têm investigado o deficit motor ao longo do desenvolvimento em crianças nascidas com MBPN, BPN ${ }^{11}$ e EBPN ${ }^{12}$. Os resultados mostraram que a permanência dos deficits motores durante a infância pode estar inversamente associada ao peso ao nascer. Por exemplo, Datar \& Jacknowitz ${ }^{11}$ não verificaram permanência dos deficits motores durante o desenvolvimento em crianças que nasceram com MBPN e BPN. Entretanto, Goyen \& Lui' ${ }^{12}$ concluíram que as crianças que nasceram com EBPN continuaram apresentando deficits motores entre as avaliações.

O desenvolvimento motor de crianças tem sido avaliado por meio de testes padronizados que são aplicados de acordo com a idade das crianças. O Movement Assessment Battery Children $(\mathrm{MABC})$ avalia habilidades motoras finas e grossas e foi criado com o objetivo de identificar crianças com transtorno do desenvolvimento da coordenação ${ }^{13}$. O Test of Gross Motor Development (TGMD) é um teste que avalia as habilidades motoras fundamentais das crianças ${ }^{14}$. Outro teste bastante utilizado é o Körperkoordination Test für Kinder (KTK), que avalia a coordenação corporal de crianças e foi criado com o objetivo de identificar crianças com problemas motores ${ }^{15}$.

Portanto, sabe-se que existe uma variabilidade entre os instrumentos de avaliação do desenvolvimento motor e a classificação do peso ao nascer. Assim, o objetivo do presente estudo foi 
analisar os estudos que associam o baixo peso ao nascer e a proficiência motora em crianças com idade entre um e dez anos.

\section{M É T O D O S}

A busca foi realizada em base de dados eletrônica (Scopus e Science Direct), e a seleção dos descritores utilizados na revisão foi feita mediante consulta aos Descritores de Assunto em Ciências da Saúde da Bireme (DECs), usando termos em português e seus correlatos em inglês. Nas buscas, foram considerados os seguintes descritores: "infant low birth weight", "infant very low birth weight", "infant extremely low birth weight", "motor skills", "psychomotor performance", "child development", "human development", "growth \& development", "growth" e "fetal development". Recorreu-se à lógica booleana para realizar a combinação dos descritores e termos utilizados para a localização das publicações. A análise dos estudos envolveu leitura de títulos, resumos e textos completos.

O critério de seleção incluiu publicações com as seguintes características: (a) artigos originais cujo tema é o baixo peso ao nascer da criança, com idade entre um e dez anos, relacionado à sua proficiência motora na infância; (b) artigos publicados entre 1990 e maio de 2011; (c) artigos originais e disponíveis na íntegra; (d) artigos que apresentassem amostra com crianças. Foram incluídos artigos que haviam sido citados nas listas de referências dos artigos selecionados que preencheram os critérios. Artigos com amostra composta por crianças deficientes foram excluídos.

Na avaliação dos artigos, foram observados os seguintes pontos: (1) faixa etária da amostra avaliada; (2) classificação do peso ao nascer (baixo peso; muito baixo peso e extremo baixo peso ao nascer); (3) instrumento utilizado na avaliação do desenvolvimento motor; (4) periódico no qual o artigo foi publicado (conforme o critério Qualis da Capes).

\section{RESULTADOS}

Mediante o cruzamento dos descritores, foram encontrados 1294 artigos (Scopus = 691 e Science Direct $=603$ ). A seleção inicial foi realizada por meio da leitura dos títulos e posterior leitura do resumo. Nessa etapa, foram escolhidos 76 artigos que poderiam atender aos critérios de inclusão. Após a avaliação dos resumos, os artigos que preenchiam todos os critérios de inclusão foram lidos na íntegra. Nessa segunda etapa, 15 artigos preencheram os critérios de inclusão estabelecidos. Durante a busca, encontrou-se um artigo publicado no ano de 1989, que foi incluído uma vez que preenchia todos os outros itens do critério de inclusão: totalizaram-se, portanto, 16 artigos. Na avaliação das listas de referências citadas nos artigos, foram localizados mais dois: assim, 18 artigos foram lidos na íntegra.

No Anexo 1, são apresentadas informações gerais sobre os 18 artigos selecionados. Destes, sete foram publicados em periódicos com Qualis "A2" na área de Medicina II16-22. Apenas dois estudos tiveram delineamento longitudinal (Datar \& Jacknowitz ${ }^{11}$; Goyen \& Lui ${ }^{12}$ ): as crianças avaliadas tinham idade entre nove meses e nove anos; cinco estudos não apresentaram grupo-controle ${ }^{11,12,18,19,23}$. Com relação ao peso de nascimento investigado, os estudos se concentram mais em crianças que apresentaram extremo baixo peso ao nascer (EBPN <1.000g) 16,17,19,20,22,24-26 e muito baixo peso ao nascer (MBPN $<1.500 \mathrm{~g})^{11,18,20,21,23,27}$ seguidos pelo baixo peso ao nascer (BPN $>1.500 \mathrm{~g})^{11,28-31}$. Apenas dois estudos (Datar \& Jacknowitz ${ }^{11}$; Keller et al. ${ }^{20}$ ) realizaram comparações entre as classificações de baixo peso ao nascer e crianças com peso normal ao nascer (PNN >2.500g).

$\mathrm{Na}$ avaliação do desenvolvimento motor, há ampla utilização de instrumentos já padronizados que avaliam habilidades motoras grossas e finas de maneira quantitativa (Anexo 2). Apesar de terem usado instrumentos padronizados que avaliam quantitativamente as habilidades motoras, Lee et al..$^{27}$ e Sommerfelt et al. ${ }^{31}$ não empregaram os escores padrão por causa da diferença 
cultural das amostras. Apenas dois estudos (Burns et al. ${ }^{25}$; Schmidhauser et al. $\left.{ }^{18}\right)$ utilizaram instrumentos que avaliam as habilidades de forma quantitativa e qualitativa. Já nos estudos de Yliherva et al. ${ }^{30}$ e Karimi et al. ${ }^{28}$, foram utilizados questionários que avaliam as habilidades motoras de forma indireta.

Os resultados dos estudos apontam que crianças nascidas com peso abaixo de $2.500 \mathrm{~g}$ apresentam algum tipo de deficit motor. As habilidades mais afetadas foram as habilidades motoras finas e o equilíbrio. Os estudos longitudinais mostraram divergência nos resultados quanto à permanência do deficit motor ao longo do desenvolvimento. Datar \& Jacknowitz ${ }^{11}$ encontraram que os efeitos do MBPN e BPN desapareceram quando as crianças foram reavaliadas aos dois anos de idade. Já os resultados encontrados por Goyen \& Lui ${ }^{12}$ apontam que as crianças continuam apresentando deficit motor quando reavaliadas aos cinco anos de idade.

\section{S C U S S Ã O}

O desenvolvimento intrauterino humano é resultante de fatores combinados como gravidez múltipla, funcionamento da placenta no útero, tamanho do corpo, idade e dieta materna ${ }^{9,32}$. Em respostas aos estímulos ambientais, o feto pode reduzir o crescimento, alterar seu metabolismo, a produção de hormônio, e sensibilidade dos tecidos aos hormônios, que podem levar a alterações nos ajustes fisiológicos e metabólicos ${ }^{9}$. Hui et al. ${ }^{33}$ verificaram que quanto menor o peso ao nascer das crianças, maior eram o crescimento na idade de zero a três meses e o índice de massa corporal aos 7 anos de idade, quando comparadas às crianças nascidas com peso normal. Batenburg-Eddes et al. ${ }^{34}$ verificaram que fetos com menor peso corporal ou com o crescimento assimétrico tinham maior probabilidade de apresentarem deficits no desenvolvimento neuromotor na infância. Portanto, a condição ambiental no início da vida tem impacto significativo em vários aspectos do desenvolvimento fetal ${ }^{32,35,36}$.
Os resultados dos estudos analisados indicam que as crianças nascidas com baixo peso apresentam deficit motor quando comparadas a crianças nascidas com peso normal. No entanto, poucos estudos fazem comparações entre as classificações de baixo peso ao nascer, a fim de verificar a severidade dos prejuízos causados, em parte, pela presença da variável baixo peso ao nascer. Os estudos que fazem comparações entre as classificações de baixo peso ao nascer indicam que quanto menor o peso ao nascer, maior é o prejuízo no desenvolvimento motor da criança.

Uma restrição metodológica encontrada nos estudos analisados é com relação à variabilidade de instrumento utilizado na avaliação da proficiência motora das crianças. De maneira geral, os estudos utilizam instrumentos já padronizados e não citam a validação dos instrumentos na população investigada, situação que pode comprometer a adequabilidade das tabelas normativas que levam ao cálculo do desempenho motor $^{37}$. Para a avaliação do desenvolvimento motor é fundamental que o avaliador conheça a dinâmica desse processo, e utilize instrumentos válidos e fidedignos para populações específi$\operatorname{cas}^{14}$. Além da avaliação quantitativa do desenvolvimento motor, é importante a realização da avaliação qualitativa dos aspectos gerais do movimento, uma vez que esse tipo de avaliação pode refletir a maturidade e a integridade do sistema nervoso central e pode ajudar no diagnóstico de transtornos de desenvolvimento ${ }^{38}$.

Outro aspecto importante a considerar é que a idade gestacional pode induzir prematuridade, que por si só é responsável pelo deficit motor. De fato, alguns outros fatores (idade gestacional, milleu hormonal, desnutrição, tabagismo, fármacos) durante a gestação podem também induzir a prematuridade e repercutir em deficit motor ao longo do desenvolvimento ${ }^{1,16,17}$. Contudo, o presente estudo tem como objetivo focar a relação existente entre o baixo peso ao nascer e o desenvolvimento motor.

Os estudos longitudinais foram controversos quanto à permanência do deficit motor 
em crianças com baixo peso ao nascer. É possível que o espectro de problemas se altere com o desenvolvimento, e mais estudos longitudinais sejam necessários para se entender esse processo $^{21}$. É importante distinguir crianças com atrasos motores daquelas com prejuízos permanentes; essa diferença tem implicações para o tratamento e para o prognóstico das crianças com baixo peso ao nascer ${ }^{24}$. Além do acompanhamento longitudinal da criança, é fundamental o conhecimento da interferência das variáveis biológicas e ambientais sobre o desenvolvimento motor.

Apenas um estudo, de caráter transversal, foi realizado no Brasil com crianças pequenas, com idade de dois anos. A variabilidade dos instrumentos, das populações e da determinação do baixo peso ao nascer dificultam a extrapolação dos resultados para as investigações realizadas no Brasil. Pouco se conhece sobre o desenvolvimento motor de crianças brasileiras nascidas com baixo peso, visto que nenhum levantamento com abrangência nacional foi realizado até o momento.

Diante dos resultados encontrados, conclui-se que quanto menor for o peso ao nascer, maiores serão os deficits provocados nas habilidades motoras básicas das crianças. No entanto, os estudos não abordam a intensidade do dano causado no desenvolvimento motor de acordo com as várias classificações de baixo peso ao nascer. Não está claro, também, se essas crianças apresentam deficits motores permanentes ao longo do desenvolvimento. Portanto, para preencher essas lacunas, é necessário que sejam realizados mais estudos longitudinais e que se utilize mais de um instrumento na avaliação motora de crianças que nascem com baixo peso.

\section{COLABORADORES}

AKM SIQUEIRA realizou a pesquisa de todos os blocos de conteúdos, a estruturação, a redação e a edição final do manuscrito. CG LEANDRO participou da orientação, da estruturação e da edição final do artigo.

\section{REFERÊ N CIAS}

1. Gluckman PD, Hanson MA. Maternal constraint of fetal growth and its consequences. Semin Fetal Neonatal Med. 2004; 9(4):419-25. doi: 10.1016/ j.siny.2004.03.001.

2. Gluckman PD, Lillycrop KA, Vickers MH, Pleasants AB, Phillips ES, Beedle AS, et al. Metabolic plasticity during mammalian development is directionally dependent on early nutritional status. Proc Natl Acad Sci USA. 2007; 104(31):12796-800. doi: 10.1073/pnas.0705667104.

3. Gluckman PD, Hanson MA, Spencer HG. Predictive adaptive responses and human evolution. Trends Ecol Evol. 2005; 20(10):527-33. doi: 10.1016/j.tree. 2005.08.001.

4. Hanson M, Godfrey KM, Lillycrop KA, Burdge GC, Gluckman PD. Developmental plasticity and developmental origins of non-communicable disease: theoretical considerations and epigenetic mechanisms. Prog Biophys Mol Biol. 2011; 106(1): 272-80. doi: 10.1016/j.pbiomolbio.2010.12.008.

5. Seckl JR, Meaney MJ. Glucocorticoid programming. Ann N Y Acad Sci. 2004; 1032:63-84. doi: 10.1196/ annals.1314.006.

6. Weinstock M. The potential influence of maternal stress hormones on development and mental health of the offspring. Brain Behav Immun. 2005; 19(4):296-308. doi: 10.1016/j.bbi.2004.09.006.

7. Oberlander TF, Warburton W, Misri S, Aghajanian J, Hertzman C. Neonatal outcomes after prenatal exposure to selective serotonin reuptake inhibitor antidepressants and maternal depression using population-based linked health data. Arch Gen Psychiatry. 2006; 63(8):898-906. doi: 10.1001/ archpsyc.63.8.898.

8. Bateson P, Barker D, Clutton-Brock T, Deb D, D'Udine B, Foley RA, et al. Developmental plasticity and human health. Nature. 2004; 430(6998): 419-21. doi: 10.1038/nature02725.

9. Gluckman PD, Hanson MA, Cooper C, Thornburg $\mathrm{KL}$. Effect of in utero and early-life conditions on adult health and disease. N Engl J Med. 2008; 359(1):61-73. doi: 10.1056/NEJMra0708473.

10. World Health Organization. Neonatal and perinatal mortality: country, regional and global estimates. Geneva: WHO; 2006.

11. Datar A, Jacknowitz $A$. Birth weight effects on children's mental, motor, and physical development: evidence from twins data. Matern Child Health J. 2009; 13(6):780-94. doi: 10.1007/ s10995-009-0461-6.

12. Goyen TA, Lui K. Longitudinal motor development of "apparently normal" high-risk infants at 18 
780 | AKM SIQUEIRA \& CG LEANDRO

months, 3 and 5 years. Early Hum Dev. 2002; 70(1-2): 103-15. doi: S0378-3782(02)00094-4.

13. Miyahara M, Tsujii M, Hanai T, Jongmans M, Barnett A, Henderson S, et al. The Movement Assessment Battery for Children: a preliminary investigation of its usefulness in Japan. Hum Mov Sci. 1998; 17(4-5): 679-97. doi: 10.1016/S0167-9457(98)00018-9.

14. Valentini N, Barbosa M, Cini G, Pick R, Spessato B, Balbinotti $M$. Test of gross motor development: expert validity, confirmatory validity and internal consistence. Rev Bras Cineantropom Hum. 2008; 10(4):399-404.

15. Smits-Engelsman B, Henderson S, Michelsen C. The assessment of children with developmental coordination disorders in the Netherlands: the relationship between the Movement Assessment Battery for Children and the Körperkoordinations Test für Kinder. Hum Mov Sci. 1998; 17(4-5): 699-709. doi: 10.1016/S0167-9457(98)00019-0.

16. Roberts G, Anderson PJ, Davis N, De Luca C, Cheong J, Doyle LW. Developmental coordination disorder in geographic cohorts of 8-year-old children born extremely preterm or extremely low birthweight in the 1990s. Dev Med Child Neurol. 2011; 53(1):55-60. doi: 10.1111/j.1469-8749.20 10.03779.x.

17. Davis NM, Ford GW, Anderson PJ, Doyle LW. Developmental coordination disorder at 8 years of age in a regional cohort of extremely-lowbirthweight or very preterm infants. Dev Med Child Neurol. 2007; 49(5):325-30. doi: 10.1111/j.1469-8 749.2007.00325.x.

18. Schmidhauser J, Caflisch J, Rousson V, Bucher HU, Largo RH, Latal B. Impaired motor performance and movement quality in very-low-birthweight children at 6 years of age. Dev Med Child Neurol. 2006; 48(9):718-22. doi: 10.1017/S001216220600154X.

19. Goyen TA, Lui K, Woods R. Visual-motor, visualperceptual, and fine motor outcomes in very-lowbirthweight children at 5 years. Dev Med Child Neurol. 1998; 40(2):76-81. doi 10.1111/j.1469-8 749.1998.tb15403.x

20. Keller H, Ayub BV, Saigal S, Bar-Or O. Neuromotor ability in 5- to 7-year-old children with very low or extremely low birthweight. Dev Med Child Neurol. 1998; 40(10):661-6. doi: 10.1111/j.1469-87 49.1998.tb12325.x.

21. Marlow N, Roberts L, Cooke R. Outcome at 8 years for children with birth weights of $1250 \mathrm{~g}$ or less. Arch Dis Child. 1993; 68(3):286-90. doi: 10.1136/ adc.68.3.

22. Marlow N, Roberts BL, Cooke RW. Motor skills in extremely low birthweight children at the age of 6 years. Arch Dis Child. 1989; 64(6):839-47.
23. Torrioli MG, Frisone MF, Bonvini L, Luciano R, Pasca MG, Lepori RT, et al. Perceptual-motor, visual and cognitive ability in very low birthweight preschool children without neonatal ultrasound abnormalities. Brain Dev. 2000; 22(3):163-8. doi: S0387-760 4(00)00098-X.

24. Holsti L, Grunau RV, Whitfield MF. Developmental coordination disorder in extremely low birth weight children at nine years. J Dev Behav Pediatr. 2002; 23(1):9-15.

25. Burns Y, Ensbey R, O'Callaghan M. Motor abilities at eight to ten years of children born weighing less than 1,000g. Physiother. 1999; 85(7):360-9. doi: 10.1016/S0031-9406(05)67189-1.

26. O'Callaghan MJ, Burns Y, Gray P, Harvey JM, Mohay $\mathrm{HI}$, Rogers $\mathrm{Y}$, et al. Extremely low birth weight and control infants at 2 years corrected age: a comparison of intellectual abilities, motor performance, growth and health. Early Hum Dev. 1995; 40(2):115-28. doi: 10.1016/0378-3782(94) 01597-I.

27. Lee SY, Chow CB, Ma PY, Ho YB, Shek CC. Gross motor skills of premature, very low-birthweight Chinese children. Ann Trop Paediatr. 2004; 24(2): 179-83. doi: 10.1179/027249304225013466.

28. Karimi M, Fallah R, Dehghanpoor A, Mirzaei M. Developmental status of 5-year-old moderate low birth weight children. Brain Dev. 2011; 33(8):651-5. doi: 10.1016/j.braindev.2010.10.022.

29. Eickmann SH, Lira PI, Lima MC. Mental and motor development at 24 months of full-term low birthweight infants. Arq Neuropsiquiatr. 2002; 60(3B):748-54.

30. Yliherva A, Olsen P, Maki-Torkko E, Koiranen M, Jarvelin MR. Linguistic and motor abilities of low-birthweight children as assessed by parents and teachers at 8 years of age. Acta Paediatr. 2001; 90(12):1440-9. doi: 10.1111/j.1651-2227.2001.tb 01611.x.

31. Sommerfelt K, Ellertsen B, Markestad T. Low birthweight and neuromotor development: a population based, controlled study. Acta Paediatr. 1996; 85(5):604-10. doi: 10.1111/j.1651-2227.1 996.tb14096.x.

32. Bateson P. Fetal experience and good adult design. Int J Epidemiol. 2001; 30(5):928-34. doi: 10.1093/ ije/30.5.928.

33. Hui LL, Schooling CM, Leung SS, Mak KH, Ho LM, Lam TH, et al. Birth weight, infant growth, and childhood body mass index: Hong Kong's children of 1997 birth cohort. Arch Pediatr Adolesc Med. 
2008; 162(3):212-8. doi: 10.1001/archpediatrics. 2007.62.

34. Batenburg-Eddes T, Groot L, Steegers EA, Hofman A, Jaddoe WW, Verhulst FC, et al. Fetal programming of infant neuromotor development: the generation R study. Pediatr Res. 2010; 67(2):132-37. doi: 10.12 03/PDR.0b013e3181c2dc76.

35. Hanson MA, Godfrey KM. Commentary: Maternal constraint is a pre-eminent regulator of fetal growth. Int J Epidemiol. 2008; 37(2):252-4. doi: 10.1093/ije/dyn015.

36. Barker DJ. The origins of the developmental origins theory. J Intern Med. 2007; 261(5):412-7. doi: 10.1 111/j.1365-2796.2007.01809.x.
37. Lopes V, Maia J, Silva R, Seabra A, Morais F. Estudo do nível de desenvolvimento da coordenação motora da população escolar (6 a 10 anos de idade) da Região Autónomados Açores. Rev Port Ciênc Desp. 2003; 3(1):47-60.

38. Kroes M, Vissers YL, Sleijpen FA, Feron FJ, Kessels $A G, B a k k e r E$, et al. Reliability and validity of a qualitative and quantitative motor test for 5 - to 6-year-old children. Eur J Paediatr Neurol. 2004; 8(3):135-43. doi: 10.1016/j.ejpn.2004.01.007.

Recebido em: 2/12/2011

Versão final em: 17/7/2012

Aprovado em: 5/9/2012 
ANEXO 1

RELAÇÃO DOS ESTUDOS QUE ASSOCIAM BAIXO PESO AO NASCER E PROFICIÊNCIA MOTORA, PUBLICADOS NO PERÍODO DE 1989 A MAIO/2011: PRIMEIRO AUTOR, PERIÓDICO, OBJETIVO DO ESTUDO, FAIXA ETÁRIA E TAMANHO DA AMOSTRA

\begin{tabular}{|c|c|c|}
\hline Primeiro autor/Periódico & Objetivo & Faixa etária/N \\
\hline $\begin{array}{l}\text { Datar \& Jacknowitz }{ }^{11} \\
\text { Maternal and Child Health Journal }\end{array}$ & $\begin{array}{l}\text { Examinar o efeito do muito baixo peso e do peso moderadamente baixo } \\
\text { ao nascer sobre o desenvolvimento motor e crescimento físico durante } \\
\text { os primeiros anos de vida de crianças }\end{array}$ & $\begin{array}{l}\text { Seguimento aos } 9 \\
\text { meses e } 2 \text { anos } \\
\text { Caso: } 6750\end{array}$ \\
\hline $\begin{array}{l}\text { Goyen \& Lui }{ }^{12} \\
\text { Early Human Development }\end{array}$ & $\begin{array}{l}\text { Examinar o desenvolvimento motor longitudinal em um grupo de crian- } \\
\text { ças de alto risco "aparentemente normais" }\end{array}$ & $\begin{array}{l}\text { Seguimento: } 18 \text { me- } \\
\text { ses, } 3 \text { e } 5 \text { anos } \\
\text { Caso: } 58\end{array}$ \\
\hline $\begin{array}{l}\text { Roberts et al. }{ }^{16} \\
\text { Developmental Medicine \& Child } \\
\text { Neurology }\end{array}$ & $\begin{array}{l}\text { Examinar a prevalência de TDC em uma coorte geográfica de crianças } \\
\text { extremamente prematuras ou com extremo baixo peso ao nascer e com- } \\
\text { parar com um grupo nascido a termo }\end{array}$ & $\begin{array}{l}8 \text { anos } \\
\text { Caso: } 189 \\
\text { Controle: } 173\end{array}$ \\
\hline $\begin{array}{l}\text { Davis et al. }{ }^{17} \\
\text { Developmental Medicine \& Child } \\
\text { Neurology }\end{array}$ & $\begin{array}{l}\text { Determinar o desempenho motor de crianças com extremo baixo peso } \\
\text { ao nascer ou muito pré-termo e comparar com crianças nascidas com } \\
\text { peso normal, estabelecer associações do TDC e suas consequências } \\
\text { cognitivas e comportamentais }\end{array}$ & $\begin{array}{l}8 \text { anos } \\
\text { Caso: } 255 \\
\text { Controle: } 208\end{array}$ \\
\hline $\begin{array}{l}\text { Schmidhauser et al. }{ }^{18} \\
\text { Developmental Medicine \& Child } \\
\text { Neurology }\end{array}$ & $\begin{array}{l}\text { Avaliar quantitativamente o desempenho motor e a qualidade dos movi- } \\
\text { mentos em crianças de muito baixo peso ao nascer }\end{array}$ & $\begin{array}{l}6 \text { anos } \\
\text { Caso: } 87\end{array}$ \\
\hline $\begin{array}{l}\text { Goyen et al. }{ }^{19} \\
\text { Developmental Medicine \& Child } \\
\text { Neurology }\end{array}$ & $\begin{array}{l}\text { Examinar a prevalência, severidade, e natureza das disfunções visual- } \\
\text { motora em um grupo de crianças com muito baixo peso e extremo baixo } \\
\text { peso ao nascer }\end{array}$ & $\begin{array}{l}5 \text { anos } \\
\text { Caso: } 83\end{array}$ \\
\hline $\begin{array}{l}\text { Keller et al. }{ }^{20} \\
\text { Developmental Medicine \& Child } \\
\text { Neurology }\end{array}$ & $\begin{array}{l}\text { Determinar se a habilidade neuromotora é prejudicada em crianças nas- } \\
\text { cidas com baixo peso }\end{array}$ & $\begin{array}{l}5 \text { a } 7 \text { anos } \\
\text { Caso: } 41 \\
\text { Controle: } 24\end{array}$ \\
\hline $\begin{array}{l}\text { Marlow et al. }{ }^{21} \\
\text { Archives of Disease in Childhood }\end{array}$ & $\begin{array}{l}\text { Examinar o desempenho educacional, motor e comportamental em } \\
\text { crianças nascidas com peso }<1.250 \mathrm{~g}\end{array}$ & $\begin{array}{l}8 \text { anos } \\
\text { Caso: } 51 \\
\text { Controle: } 36\end{array}$ \\
\hline $\begin{array}{l}\text { Marlow et al. }{ }^{22} \\
\text { Archives of Disease in Childhood }\end{array}$ & $\begin{array}{l}\text { Verificar se crianças nascidas com extremo baixo peso apresentam pre- } \\
\text { juízos nas habilidades motoras quando comparadas com crianças nasci- } \\
\text { das com peso normal }\end{array}$ & $\begin{array}{l}6 \text { anos } \\
\text { Caso: } 53 \\
\text { Controle: } 53\end{array}$ \\
\hline $\begin{array}{l}\text { Torrioli et al. }{ }^{23} \\
\text { Brain \& Development }\end{array}$ & $\begin{array}{l}\text { Fornecer informações adicionais sobre o desenvolvimento perceptual- } \\
\text { motor e cognitivo, bem como padrões comportamentais de crianças na } \\
\text { idade pré-escolar com muito baixo peso ao nascer }\end{array}$ & $\begin{array}{l}\text { Média de } 4,9 \text { anos } \\
\text { Caso: } 36 \text { crianças }\end{array}$ \\
\hline $\begin{array}{l}\text { Holsti et al. }{ }^{24} \\
\text { Developmental and Behavioral } \\
\text { Pediatrics }\end{array}$ & $\begin{array}{l}\text { Descrever a prevalência de TDC em uma coorte de crianças nascidas } \\
\text { com extremo baixo peso ao nascer e comparar as dificuldades acadêmi- } \\
\text { cas associadas em crianças com extremo baixo peso ao nascer com e } \\
\text { sem TDC }\end{array}$ & $\begin{array}{l}9 \text { anos } \\
\text { Caso: } 73 \\
\text { Controle: } 18\end{array}$ \\
\hline $\begin{array}{l}\text { Burns et al. }{ }^{25} \\
\text { Physiotherapy }\end{array}$ & $\begin{array}{l}\text { Investigar os tipos de problemas motores em crianças nascidas com ex- } \\
\text { tremo baixo peso }\end{array}$ & $\begin{array}{l}\text { Média de } 8,99 \text { anos } \\
\text { Caso: } 29 \\
\text { Controle: } 12\end{array}$ \\
\hline $\begin{array}{l}\text { O'Callaghan et al. }{ }^{26} \\
\text { Early Human Development }\end{array}$ & $\begin{array}{l}\text { Comparar desenvolvimento, habilidades motoras, crescimento e saúde } \\
\text { de crianças nascidas com extremo baixo peso com crianças nascidas com } \\
\text { peso normal }\end{array}$ & $\begin{array}{l}2 \text { anos } \\
\text { Caso: } 63 \\
\text { Controle: } 44\end{array}$ \\
\hline $\begin{array}{l}\text { Lee et al. }{ }^{27} \\
\text { Annals of Tropical Paediatrics }\end{array}$ & $\begin{array}{l}\text { Investigar se crianças asiáticas prematuras, muito baixo peso ao nascer e } \\
\text { sem grandes prejuízos, tem habilidades motoras pobres em comparação } \\
\text { com crianças nascidas com peso normal }\end{array}$ & $\begin{array}{l}\text { Entre } 5 \text { e } 7 \text { anos } \\
\text { Caso: } 42 \\
\text { Controle: } 69\end{array}$ \\
\hline $\begin{array}{l}\text { Karimi et al. }{ }^{28} \\
\text { Brain \& Development }\end{array}$ & $\begin{array}{l}\text { Avaliar e comparar o estado de desenvolvimento das crianças modera- } \\
\text { damente baixo peso ao nascer com as crianças nascidas com peso nor- } \\
\text { mal }\end{array}$ & $\begin{array}{l}5 \text { anos } \\
\text { Caso: } 150 \\
\text { Controle: } 150\end{array}$ \\
\hline $\begin{array}{l}\text { Eickmann et al. }{ }^{29} \\
\text { Arquivos de Neuropsiquiatra }\end{array}$ & $\begin{array}{l}\text { Comparar o desenvolvimento mental e motor de crianças nascidas a } \\
\text { termo com baixo peso e peso adequado }\end{array}$ & $\begin{array}{l}2 \text { anos } \\
\text { Caso: } 76 \\
\text { Controle: } 76\end{array}$ \\
\hline $\begin{array}{l}\text { Yliherva et al. }{ }^{30} \\
\text { Acta Paediatrica }\end{array}$ & $\begin{array}{l}\text { Estudar as habilidades motoras e linguísticas de crianças nascidas com } \\
\text { baixo peso }\end{array}$ & $\begin{array}{l}8 \text { anos } \\
\text { Caso: } 279 \\
\text { Controle: } 8091\end{array}$ \\
\hline $\begin{array}{l}\text { Sommerfelt et al. }{ }^{31} \\
\text { Acta Paediatrica }\end{array}$ & $\begin{array}{l}\text { Investigar a relação entre baixo peso ao nascer e o desenvolvimento motor } \\
\text { em pré-escolares e o valor preditivo de fatores pré, peri e neonatal para } \\
\text { o desenvolvimento neuromotor }\end{array}$ & $\begin{array}{l}5 \text { anos } \\
\text { Caso: } 144 \\
\text { Controle: } 163\end{array}$ \\
\hline
\end{tabular}

TDC: Transtorno do Desenvolvimenro da Coordenação. 


\title{
ANEXO 2
}

\begin{abstract}
RELAÇ̃̃O DOS ESTUDOS QUE ASSOCIAM BAIXO PESO AO NASCER E PROFICIÊNCIA MOTORA, PUBLICADOS NO PERÍODO DE 1989 A MAIO/2011: PRIMEIRO AUTOR, PESO DE NASCIMENTO, INSTRUMENTOS DE AVALIAÇÃO DO DESENVOLVIMENTO MOTOR E RESULTADOS
\end{abstract}

\begin{tabular}{|c|c|c|c|}
\hline Primeiro autor & Peso de nascimento & $\begin{array}{l}\text { Instrumentos de avaliação do } \\
\text { desenvolvimento motor }\end{array}$ & Resultados principais \\
\hline Datar \& Jacknowitz ${ }^{11}$ & $\begin{array}{l}\text { MBPN: }<1.500 \mathrm{~g} \\
\text { BPN: } 1.500 \mathrm{~g} \text { a } 2.499 \mathrm{~g} \\
\text { PNN: }>2.500 \mathrm{~g}\end{array}$ & $\begin{array}{l}\text { DM: Bayley Short Form - Research Edition } \\
\text { (BSF-R) }\end{array}$ & $\begin{array}{l}\text { O MBPN e BPN tem efeitos sobre o DM aos } \\
9 \text { meses de idade. Aos } 2 \text { anos de idade os } \\
\text { efeitos do MBPN e BPN são insignificantes }\end{array}$ \\
\hline Goyen et al. ${ }^{12}$ & EBPN $:<1.000 \mathrm{~g}$ & $\begin{array}{l}\text { DM: Peabody Developmental Motor } \\
\text { Scales }\end{array}$ & $\begin{array}{l}\text { O quociente do DM grosso diminuiu ao lon- } \\
\text { go do tempo ( } 18 \text { meses, } 3 \text { e } 5 \text { anos). } 63,8 \% \\
\text { continuaram a ter déficit motor fino aos } 5 \\
\text { anos de idade }\end{array}$ \\
\hline Roberts et al. ${ }^{16}$ & $\begin{array}{l}\text { EBPN: } 500 \mathrm{~g} \text { a } 999 \mathrm{~g} \\
\text { PNN: }>2.500 \mathrm{~g}\end{array}$ & $\begin{array}{l}\text { DM: Movement Assessment Battery for } \\
\text { Children (MABC) }\end{array}$ & $\begin{array}{l}\text { Prejuízo motor leve a moderado foi mais } \\
\text { comum em crianças com EBPN }\end{array}$ \\
\hline Davis et al. ${ }^{17}$ & $\begin{array}{l}\text { EBPN }:<1.000 \mathrm{~g} \\
\text { PNN }:>2.500 \mathrm{~g}\end{array}$ & $\begin{array}{l}\text { DM: Movement Assessment Battery for } \\
\text { Children (MABC) }\end{array}$ & $\begin{array}{l}\text { As crianças de EBPN apresentam desempe- } \\
\text { nho inferior nas habilidades motoras em re- } \\
\text { lação às crianças de PNN }\end{array}$ \\
\hline Schmidhauser et al. ${ }^{18}$ & MBPN: $<1.250 \mathrm{~g}$ & DM: Zurich Neuromotor Assessment & $\begin{array}{l}\text { O desempenho neuromotor das crianças de } \\
\text { MBPN foi menor em todos os componen- } \\
\text { tes cronometrados. A tarefa motora fina } \\
\text { adaptativa fina (pegboard) e o equilíbrio fo- } \\
\text { ram particularmente afetados }\end{array}$ \\
\hline Goyen et al. ${ }^{19}$ & EBPN $:<1.000 \mathrm{~g}$ & $\begin{array}{l}\text { DM: Peabody Developmental Motor } \\
\text { Scales }\end{array}$ & $\begin{array}{l}\text { As crianças com EBPN tinham escores abaixo } \\
\text { da média para a função motora fina }\end{array}$ \\
\hline Keller et al. ${ }^{20}$ & $\begin{array}{l}\text { EBPN: } 500 \mathrm{~g} \text { a } 999 \mathrm{~g} \\
\text { MBPN: } 1.000 \mathrm{~g} \text { a } 1.499 \mathrm{~g} \\
\text { PNN: }>2.500 \mathrm{~g}\end{array}$ & $\begin{array}{l}\text { DM: Körperkoordinations- test für Kinder } \\
\text { (KTK) }\end{array}$ & $\begin{array}{l}\text { As crianças com EBPN tiveram menor desem- } \\
\text { penho motor quando comparadas com os } \\
\text { outros grupos }\end{array}$ \\
\hline Marlow et al. ${ }^{21}$ & MBPN: $<1.250 \mathrm{~g}$ & DM: Test of Motor Impairment & $\begin{array}{l}\text { As crianças com MBPN apresentaram maior } \\
\text { comprometimento motor }\end{array}$ \\
\hline Marlow et al. ${ }^{22}$ & EBPN: $<1.250 \mathrm{~g}$ & DM: Test of Motor Impairment & $\begin{array}{l}\text { As crianças com EBPN foram mais prejudi- } \\
\text { cadas no teste motor que as crianças do gru- } \\
\text { po controle }\end{array}$ \\
\hline Torrioli et al..$^{23}$ & MBPN: $<1.500 \mathrm{~g}$ & $\begin{array}{l}\text { DM: Movement Assessment Battery for } \\
\text { Children (MABC) }\end{array}$ & $\begin{array}{l}\text { A maioria das crianças com MBPN apresen- } \\
\text { tou escores anormais ou limítrofes, portan- } \\
\text { to, eram considerados como tendo proble- } \\
\text { ma perceptual-motor }\end{array}$ \\
\hline Holsti et al. ${ }^{24}$ & MBPN: <1.500g & $\begin{array}{l}\text { DM: Bruininks Oseretsky Test of Motor } \\
\text { Proficiency (BOMP) }\end{array}$ & $\begin{array}{l}\text { As crianças classificadas com TDC tinham } \\
\text { baixo escore na composição motora grossa, } \\
\text { na composição motora fina em ambas as } \\
\text { composições grossas e finas }\end{array}$ \\
\hline Burns et al. ${ }^{25}$ & EBPN: $<1.000 \mathrm{~g}$ & DM: 30 Minute Battery & $\begin{array}{l}\text { As crianças de EBPN continuaram mostra- } \\
\text { ram desempenho inferior no equilíbrio so- } \\
\text { bre uma perna e habilidade motora fina para } \\
\text { escrever, quando comparadas com as crian- } \\
\text { ças de PNN }\end{array}$ \\
\hline $\mathrm{O}^{\prime}$ Callaghan et al. ${ }^{26}$ & EBPN: $<1.000 \mathrm{~g}$ & $\begin{array}{l}\text { DM: Neurosensory Motor } \\
\text { Developmental Assessment (NSMDA) }\end{array}$ & $\begin{array}{l}\text { As crianças de EBPN foram diferentes do gru- } \\
\text { po controle na pontuação do NSMDA nas } \\
\text { habilidades motoras grossas e finas }\end{array}$ \\
\hline Lee et al. ${ }^{27}$ & MBPN: <1.500g & $\begin{array}{l}\text { DM: Peabody Developmental Motor } \\
\text { Scales (PDMS) }\end{array}$ & $\begin{array}{l}\text { O grupo MBPN teve escores mais baixos em } \\
\text { habilidades de equilíbrio e não locomotoras. } \\
\text { O escore total do teste também foi menor }\end{array}$ \\
\hline
\end{tabular}


784 | AKM SIQUEIRA \& CG LEANDRO

ANEXO 2

RELAÇÃO DOS ESTUDOS QUE ASSOCIAM BAIXO PESO AO NASCER E PROFICIÊNCIA MOTORA, PUBLICADOS

NO PERÍODO DE 1989 A MAIO/2011: PRIMEIRO AUTOR, PESO DE NASCIMENTO, INSTRUMENTOS DE AVALIAÇÃO DO DESENVOLVIMENTO MOTOR E RESULTADOS

\begin{tabular}{|c|c|c|c|}
\hline Primeiro autor & Peso de nascimento & $\begin{array}{l}\text { Instrumentos de avaliação do } \\
\text { desenvolvimento motor }\end{array}$ & Resultados principais \\
\hline Karimi et al. ${ }^{28}$ & $\begin{array}{l}\text { BPN: } 1.500 \mathrm{~g} \text { a } 2.499 \mathrm{~g} \\
\text { PNN: } 2.500 \mathrm{~g} \text { a } 4.000 \mathrm{~g}\end{array}$ & $\begin{array}{l}\text { Desenvolvimento motor (DM): } \\
\text { Ages and Stages Questionnaires (ASQ) }\end{array}$ & $\begin{array}{l}\text { As crianças com BPN tinham mais proble- } \\
\text { mas no desenvolvimento motor grosso e } \\
\text { fino }\end{array}$ \\
\hline Eickmann et al..$^{29}$ & BPN: $1.500 \mathrm{~g}$ a $2.499 \mathrm{~g}$ & DM: Bayley Scale of Infant Development & $\begin{array}{l}\text { As crianças de BPN apresentam DM mais } \\
\text { baixo que as crianças do grupo controle }\end{array}$ \\
\hline Yliherva et al. ${ }^{30}$ & $\begin{array}{l}\text { BPN: }<2.500 \mathrm{~g} \\
\text { PNN }:>2.500 \mathrm{~g}\end{array}$ & $\begin{array}{l}\text { DM: Questionário construído especial- } \\
\text { mente para o estudo }\end{array}$ & $\begin{array}{l}\text { As crianças de BPN tinham mais problemas } \\
\text { que as crianças PNN nas habilidades motoras } \\
\text { finas, habilidades motoras grossas e coorde- } \\
\text { nação }\end{array}$ \\
\hline Sommerfelt et al. ${ }^{31}$ & $\begin{array}{l}\text { BPN: }<2.000 \mathrm{~g} \\
\text { PNN: }>3.000 \mathrm{~g}\end{array}$ & $\begin{array}{l}\text { DM: Peabody Developmental Motor } \\
\text { Scales (PDMS) }\end{array}$ & $\begin{array}{l}\text { Os meninos com BPN tinham mais pontua- } \\
\text { ções anormais no equilíbrio que os meninos } \\
\text { de PNN }\end{array}$ \\
\hline
\end{tabular}

DM: Desenvolvimento Motor; ASQ: Ages and Stages Questionnaires; MABC: Movement Assessment Battery for Children; BSF-R: Bayley Short Form - Research Edition; PDMS: Peabody Developmental Motor Scales; BOMP: Bruininks Oseretsky Test für Kinder; NSMDA: Neurosensory Motor Developmental Assessment; EBPN: Extremo Baixo Peso ao Nascer. 